\title{
A Family of Minimal Surfaces and Univalent Planar Harmonic Mappings
}

\author{
Michael Dorff ${ }^{1}$ and Stacey Muir ${ }^{2}$ \\ ${ }^{1}$ Department of Mathematics, Brigham Young University, Provo, UT 84602, USA \\ ${ }^{2}$ Department of Mathematics, University of Scranton, Scranton, PA 18510, USA \\ Correspondence should be addressed to Stacey Muir; stacey.muir@scranton.edu
}

Received 30 December 2013; Accepted 22 January 2014; Published 3 April 2014

Academic Editor: Rosihan M. Ali

Copyright (c) 2014 M. Dorff and S. Muir. This is an open access article distributed under the Creative Commons Attribution License, which permits unrestricted use, distribution, and reproduction in any medium, provided the original work is properly cited.

\begin{abstract}
We present a two-parameter family of minimal surfaces constructed by lifting a family of planar harmonic mappings. In the process, we use the Clunie and Sheil-Small shear construction for planar harmonic mappings convex in one direction. This family of minimal surfaces, through a continuous transformation, has connections with three well-known surfaces: Enneper's surface, the wavy plane, and the helicoid. Moreover, the identification process used to recognize the surfaces provides a connection to surfaces that give tight bounds on curvature estimates first studied in a 1988 work by Hengartner and Schober.
\end{abstract}

\section{Introduction}

A recent approach to investigate minimal surfaces in $\mathbb{R}^{3}$ has utilized results about harmonic mappings in the plane [1-7]. Specifically, the Weierstrass-Enneper representation which provides a formula for the local representation of a minimal surface in $\mathbb{R}^{3}$ is used, but in most cases the authors have been unable to identify the minimal graphs constructed from the corresponding planar harmonic mapping. For example, in [5], Hengartner and Schober found curvature estimates for any minimal surface lying over a strip domain, a half-plane, and a slit plane with one slit lying on the real axis. For each domain, they found surfaces for which the estimates were sharp; however, they were only able to identify the surface providing the tight bounds for one of the three domains. In this paper, we identify the remaining two surfaces. Moreover, we construct a family of minimal graphs associated with a family of harmonic mappings built through the Clunie and Sheil-Small shear construction [8]. This process leads to a connection between three well-known surfaces: the Enneper surface, the wavy plane, and the helicoid. Using conjugate surfaces provides a further identification method between harmonic mappings and minimal surfaces. In doing so, we extend the family mentioned above to a two-parameter family of minimal graphs.

\section{Background}

We begin with background on harmonic functions defined on the open unit disk $\mathbb{D}=\{z \in \mathbb{C}:|z|<1\}$. Let $\mathscr{H}_{0}(\mathbb{D})$ be the set of analytic functions on $\mathbb{D}$ that fix zero. Define $S$ as the set of functions $f \in \mathscr{H}_{0}(\mathbb{D})$ which are univalent and have the additional normalization $f^{\prime}(0)=1$. A harmonic function $f: \mathbb{D} \rightarrow \mathbb{C}$ with $f(0)=0$ can be uniquely represented as $f=h+\bar{g}$ with $h, g \in \mathscr{H}_{0}(\mathbb{D})$. Furthermore, if we write $f(z)=$ $f(x+i y)=u(x, y)+i v(x, y)$, then $f$ is sense preserving if the Jacobian, $J_{f}$, of the mapping $(x, y) \mapsto(u, v)$ is positive. The function $f$ is locally univalent if $J_{f}$ never vanishes in $\mathbb{D}$. By a result of Lewy [9], $f=h+\bar{g}$ is locally univalent and sense preserving if and only if $\left|g^{\prime}(z)\right|<\left|h^{\prime}(z)\right|$ for all $z \in \mathbb{D}$. In this case, we simply say $f$ is locally univalent. In addition, we call $f$ univalent if $f$ is one-to-one and sense preserving in $\mathbb{D}$. Let $S_{H}^{0}$ be the family of harmonic univalent functions on $\mathbb{D}$ of the form $f=h+\bar{g}$ with $h(0)=g(0)=g^{\prime}(0)=0$ and $h^{\prime}(0)=1$. Clearly $S \mp S_{H}^{0}$. The dilatation of $f \in S_{H}^{0}$ is the function $\omega: \mathbb{D} \rightarrow \mathbb{C}$ given by $\omega(z)=g^{\prime}(z) / h^{\prime}(z)$.

We recall that a domain $D$ is convex in the direction of $\varphi$, $\varphi \in[0, \pi)$, if every line parallel to the line through 0 and $e^{i \varphi}$ has a connected intersection with $D$, and a domain $D$ that is convex in every direction is convex. If $\varphi=0$, we say that $D$ is convex in the direction of the real axis. The following theorem 
due to Clunie and Sheil-Small [8] is often referred to as the shear construction and will be critical to our minimal surface constructions.

Theorem 1. Let $\varphi \in[0, \pi)$. A harmonic $f=h+\bar{g}$ locally univalent in $\mathbb{D}$ is a univalent mapping of $\mathbb{D}$ onto a domain convex in the direction of $\varphi$ if and only if $h-e^{2 i \varphi} g$ is a conformal univalent mapping of $\mathbb{D}$ onto a domain convex in the direction of $\varphi$.

We now turn to some background on minimal surfaces prior to introducing the connection between harmonic mappings and minimal surfaces.

A regular surface $S \subseteq \mathbb{R}^{3}$ can be parameterized by a map $\mathbf{X}: \Omega \subseteq \mathbb{R}^{2} \rightarrow \mathbb{R}^{3}$ written as $\mathbf{X}(u, v)=\left(x_{1}(u, v), x_{2}(u, v)\right.$, $\left.x_{3}(u, v)\right)$. At a given point $P_{0}=\mathbf{X}\left(u_{0}, v_{0}\right)$, there exists a normal vector $\mathbf{n}$. Let $\mathbf{T}$ be a tangent direction at $P_{0}$ and let $C$ be the curve of intersection of the surface and the plane containing $\mathbf{n}$ and $\mathbf{T}$. Letting $\alpha(s)$ be the parameterization of $C$ with respect to arclength $s$, the normal curvature in the direction $T$ is given by $k(\mathbf{T})=\alpha^{\prime \prime}(s) \cdot \mathbf{n}$ where we have assumed the surface has continuous second partial derivatives in its parametric representations. The principal curvatures $k_{1}$ and $k_{2}$ of the surface at $P_{0}$ are the maximum and minimum values of $k(\mathbf{T})$ as $\mathbf{T}$ ranges over all directions in the tangent plane. The mean curvature of the surface at $P_{0}$ is $H=\left(k_{1}+k_{2}\right) / 2$ and a surface is minimal if $H \equiv 0$ at every point on the surface. Geometrically, this means that the principal curvatures bend to the same extent in opposite directions with respect to the normal. Note that we will use the term minimal graph when a minimal surface has no self-intersections over its given domain. More about minimal surfaces can be found in [10-12].

One classical way to connect two minimal surfaces is through what are called conjugate surfaces. Let $\mathbf{X}$ and $\mathbf{Y}$ be parameterizations of surfaces from a region $\Omega \subsetneq \mathbb{C}$ into $\mathbb{R}^{3}$. Then $\mathbf{X}$ and $\mathbf{Y}$, given in terms of $z=u+i v \in \Omega$, are conjugate if they satisfy the Cauchy-Riemann equations. That is, they satisfy $\mathbf{X}_{u}=\mathbf{Y}_{v}$ and $\mathbf{X}_{v}=-\mathbf{Y}_{u}$. Additionally, any two conjugate minimal surfaces $\mathbf{X}$ and $\mathbf{Y}$ can be joined through the one-parameter family of minimal surfaces given by

$$
\mathbf{Z}=\cos t \mathbf{X}+\sin t \mathbf{Y}, \quad t \in \mathbb{R} .
$$

Clearly, when $t=0$, we have the minimal surface parameterized by $\mathbf{X}$ and when $t=\pi / 2$ we have the minimal surface parameterized by $\mathbf{Y}$. Thus, for $0 \leq t \leq \pi / 2$, we have a family of minimal surfaces known as associated surfaces.

Three minimal surfaces that will arise in this paper are the helicoid, wavy plane, and Enneper's surface. The helicoid can be parameterized on $\mathbb{D} \backslash(-1,0]$ in the following way:

$$
\mathbf{Y}_{0}(z)=\left(\operatorname{Re}\left(z-\frac{1}{z}\right), \operatorname{Im}\left(z+\frac{1}{z}\right), 2 \operatorname{Im} \log z\right) .
$$

The wavy plane can be parameterized on $\mathbb{D} \backslash(-1,0]$ by

$$
\begin{aligned}
\mathbf{Y}_{1}(z)=( & \frac{1}{2} \operatorname{Re}\left(\log z-\frac{1}{2} z^{2}\right),-\frac{1}{2} \operatorname{Im}\left(\log z+\frac{1}{2} z^{2}\right), \\
& \operatorname{Im}(i z)) .
\end{aligned}
$$

Enneper's surface can be parameterized on $\mathbb{D}$ by

$$
\mathbf{Y}_{2}(z)=\left(\operatorname{Re}\left(z-\frac{1}{3} z^{3}\right), \operatorname{Im}\left(z+\frac{1}{3} z^{3}\right), \operatorname{Im}\left(-i z^{2}\right)\right) .
$$

It is worth noting that scalings and reflections across planes containing two axes do not alter the geometry of minimal surfaces.

In this paper, we will use the shear construction (Theorem 1) to produce a family of harmonic mappings that can be lifted to a family of minimal surfaces by using the following version of Weierstrass-Enneper representation (cf. [13, pp. 177-178]).

Theorem 2 (Weierstrass-Enneper representation). Let $\Omega \mp$ $\mathbb{C}$ be a simply connected domain containing the origin. If a minimal graph

$$
\{(u, v, F(u, v)): u+i v \in \Omega\}
$$

is parameterized by sense-preserving isothermal parameters $z=x+i y \in \mathbb{D}$, the projection onto its base plane defines a harmonic mapping $w=u+i v=f(z)$ of $\mathbb{D}$ onto $\Omega$ whose dilatation is the square of an analytic function. Conversely, if $f=h+\bar{g}$ is a harmonic univalent mapping of $\mathbb{D}$ onto $\Omega$ with dilatation $\omega=g^{\prime} / h^{\prime}$ being the square of an analytic function, then with $z=x+i y \in \mathbb{D}$, the parameterization

$$
\begin{aligned}
\mathbf{X}(z)=( & \operatorname{Re}\{h(z)+g(z)\}, \operatorname{Im}\{h(z)-g(z)\}, \\
& \left.2 \operatorname{Im}\left\{\int_{0}^{z} \sqrt{g^{\prime}(\zeta) h^{\prime}(\zeta)} d \zeta\right\}\right)
\end{aligned}
$$

defines a minimal graph whose projection into the complex plane is $f(\mathbb{D})$. Except for the choice of sign and an arbitrary additive constant in the third coordinate function, this is the only such surface.

While the Weierstrass-Enneper representation theorem states that a univalent harmonic map lifts to a minimal surface that is a graph, a harmonic map that has a dilatation that is a perfect square always lifts to a minimal surface but there is no guarantee that the surface is a graph.

\section{A Family of Minimal Surfaces}

In this section, we use the shear construction derived from Theorem 1 to build a family of harmonic univalent functions with a range convex in one direction that lifts to a family of minimal graphs as described in Theorem 2. Moreover, we identify precisely which minimal graph the function lifts to for various members of this family and provide a connection between several well-known minimal surfaces. In doing so, we will identify the surfaces utilized by Hengartner and Schober in [5] to show tightness for bounds on curvature estimates for surfaces lying over a half-plane and a slit plane with one slit on the negative real axis. Finally, we use a result on conjugate surfaces to introduce a two-parameter family of minimal surfaces. 
Before we introduce the family of harmonic mappings, we need some background on the analytic function commonly referred to as the generalized Koebe function. For $c \in[0,2]$, define $k_{c}: \mathbb{D} \rightarrow \mathbb{C}, c \in[0,2]$, as

$$
k_{c}(z)=\int_{0}^{z} \frac{(1+w)^{c-1}}{(1-w)^{c+1}} d w \text {. }
$$

We easily see

$$
\begin{gathered}
k_{0}(z)=\frac{1}{2} \log \frac{1+z}{1-z}, \\
k_{c}(z)=\frac{1}{2 c}\left(\left(\frac{1+z}{1-z}\right)^{c}-1\right), \quad c \in(0,2] .
\end{gathered}
$$

Notice $k_{1}(z)=z /(1-z)$ and $k_{2}(z)=z /(1-z)^{2}$. For $c \in[0,2]$, $k_{c} \in S$ and $k_{c}(\mathbb{D})$ is convex in the direction of the real axis. Additionally, for $c \in[0,1], k_{c}(\mathbb{D})$ is convex. From this, we have the following theorem.

Theorem 3. For $c \in[0,2]$, define $f_{c}=h_{c}+\overline{g_{c}}: \mathbb{D} \rightarrow \mathbb{C}$ to be the harmonic mapping satisfying

$$
h_{c}(z)-g_{c}(z)=k_{c}(z), \quad g_{c}^{\prime}(z)=z^{2} h_{c}^{\prime}(z)
$$

normalized by $h_{c}(0)=g_{c}(0)=g_{c}^{\prime}(0)=h_{c}^{\prime}(0)-1=0$ where $k_{c}$ is given by (7). Then $f_{c} \in S_{H}^{0}$ and $f_{c}(\mathbb{D})$ is convex in the direction of the real axis, and as $c$ varies from 0 to $2, f_{c}(\mathbb{D})$ transforms from a strip mapping to a slit mapping.

Proof. By Theorem 1, $f_{c} \in S_{H}^{0}$ and $f_{c}(\mathbb{D})$ is convex in the direction of the real axis. What remains to be shown are the mapping properties of $f_{c}$.

Using (9) to solve for $h_{c}$ and $g_{c}$ gives

$h_{c}(z)$

$$
= \begin{cases}\frac{1}{4}\left(\frac{2 z}{1-z^{2}}+\log \left(\frac{1+z}{1-z}\right)\right), & \text { if } c=0, \\ \frac{1}{4}\left(\frac{3 z-2 z^{2}}{(1-z)^{2}}+\frac{1}{2} \log \left(\frac{1+z}{1-z}\right)\right), & \text { if } c=1, \\ \frac{1}{4}\left(\frac{1}{c\left(1-c^{2}\right)}\left(\frac{1+z}{1-z}\right)^{c}\right. & \\ \left.\quad \times \frac{1-2 c^{2}+2 c z-z^{2}}{1-z^{2}}+\frac{2 c^{2}-1}{c\left(1-c^{2}\right)}\right), & \text { if } c \neq 0,1,\end{cases}
$$

$g_{c}(z)$

$$
= \begin{cases}\frac{1}{4}\left(\frac{2 z}{1-z^{2}}-\log \left(\frac{1+z}{1-z}\right)\right), & \text { if } c=0, \\ \frac{1}{4}\left(\frac{-z+2 z^{2}}{(1-z)^{2}}+\frac{1}{2} \log \left(\frac{1+z}{1-z}\right)\right), & \text { if } c=1, \\ \frac{1}{4}\left(\frac{1}{c\left(1-c^{2}\right)}\left(\frac{1+z}{1-z}\right)^{c}\right. & \\ \left.\quad \times \frac{-1+2 c z+\left(1-2 c^{2}\right) z^{2}}{1-z^{2}}+\frac{1}{c\left(1-c^{2}\right)}\right), & \text { if } c \neq 0,1 .\end{cases}
$$

In order to understand the mapping properties of $f_{c}$, we perform a change of variables using

$$
w=\frac{1+z}{1-z} .
$$

With $z=(w-1) /(w+1)$, this substitution leads to

$$
\begin{aligned}
& h_{c}(z)= \begin{cases}\frac{1}{8}\left(2 \log w-\frac{1}{w}+w\right), & \text { if } c=0, \\
\frac{1}{8}\left(\frac{1}{2} w^{2}+2 w+\log w-\frac{5}{2}\right), & \text { if } c=1, \\
\frac{1}{8}\left(\frac{1}{c+1} w^{c+1}+\frac{2}{c} w^{c}+\frac{1}{c-1} w^{c-1}\right. & \\
\left.-\frac{2\left(2 c^{2}-1\right)}{c\left(c^{2}-1\right)}\right), & \text { if } c \neq 0,1,\end{cases} \\
& g_{c}(z)= \begin{cases}\frac{1}{8}\left(-2 \log w-\frac{1}{w}+w\right), & \text { if } c=0, \\
\frac{1}{8}\left(\frac{1}{2} w^{2}-2 w+\log w+\frac{3}{2}\right), & \text { if } c=1, \\
\frac{1}{8}\left(\frac{1}{c+1} w^{c+1}-\frac{2}{c} w^{c}+\frac{1}{c-1} w^{c-1}\right. & \text { if } c \neq 0,1 .\end{cases}
\end{aligned}
$$

By writing $w=x+i y, x>0$ and $y \in \mathbb{R}$, and $f_{c}(z)=u_{c}+i v_{c}$, again with $z=(w-1) /(w+1)$, it is not difficult to show that $f_{0}$ maps $\mathbb{D}$ onto the strip $\{z \in \mathbb{C}:|\operatorname{Im} z|<\pi / 4\}$ and $f_{2}$ maps $\mathbb{D}$ onto $\mathbb{C} \backslash\{z \in \mathbb{C}: z<-1 / 3\}$.

See Figure 1 for graphs of $f_{c}(|z|=r)$ for various values of $c \in[0,2]$ and $r<1$. It is worth noting that by the above work, we may also explicitly describe the image of $f_{1}(\mathbb{D})$ to be the region containing the origin and bounded by the curve $u=1 / 8\left(-4 v^{2}+2 \ln (2|v|)-1\right)$ in the $u v$-plane.

We are now prepared to introduce a family of minimal graphs which will later be extended to the two-parameter family of minimal graphs discussed in the introduction.

Theorem 4. For $c \in[0,2]$, define $f_{c}=h_{c}+\overline{g_{c}}: \mathbb{D} \rightarrow \mathbb{C}$ to be the harmonic mapping satisfying $(9)$ normalized by $h_{c}(0)=$ $g_{c}(0)=g_{c}^{\prime}(0)=h_{c}^{\prime}(0)-1=0$. Then $f_{c}$ lifts to a minimal graph $\mathbf{X}_{c}$ on $\mathbb{D}$ for each $c \in[0,2]$. Moreover, $\mathbf{X}_{0}(\mathbb{D})$ is a part of the helicoid, $\mathbf{X}_{1}(\mathbb{D})$ is a part of the wavy plane, and $\mathbf{X}_{2}(\mathbb{D})$ is a part of the Enneper surface.

Proof. Since $g_{c}^{\prime}(z)=z^{2} h_{c}^{\prime}(z)$, by Theorem 2, $f_{c}$ lifts to a minimal graph on $\mathbb{D}$ for each $c \in[0,2]$. Applying this theorem yields the following representations of minimal graphs for $c=0,1,2$ :

$$
\mathbf{X}_{0}(z)=\left(\operatorname{Re} \frac{z}{1-z^{2}}, \frac{1}{2} \operatorname{Im}\left(\log \frac{1+z}{1-z}\right), \operatorname{Im} \frac{z^{2}}{1-z^{2}}\right),
$$




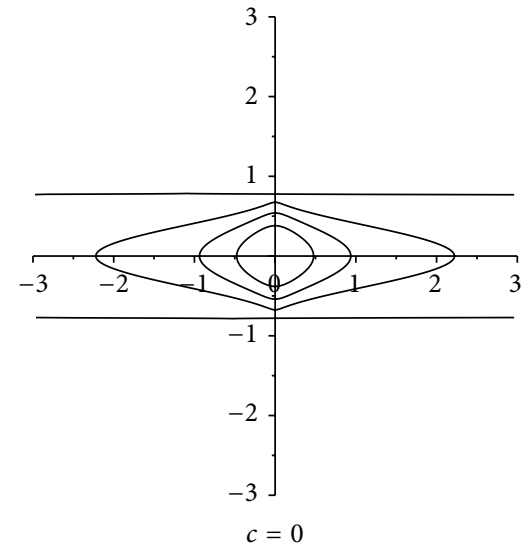

(a)

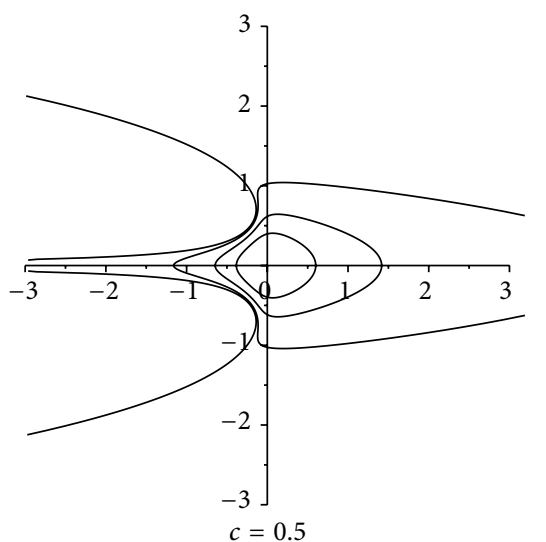

(c)

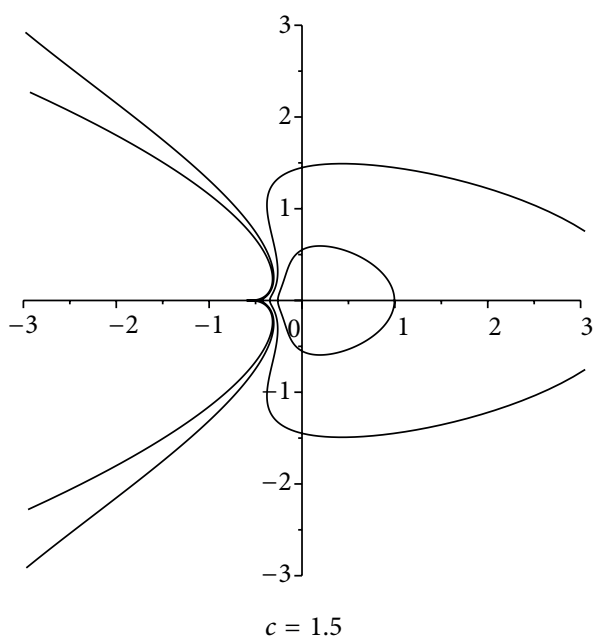

(e)

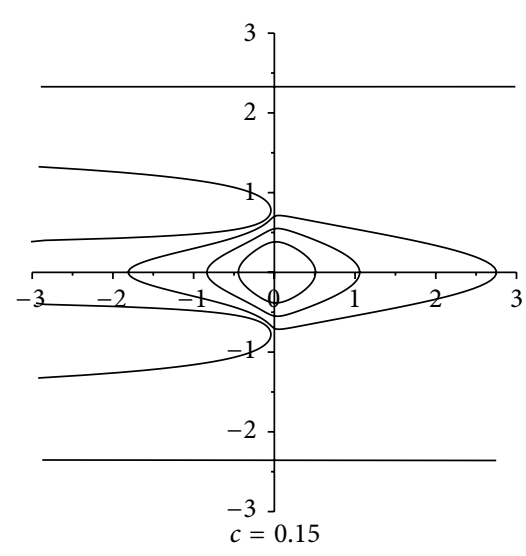

(b)

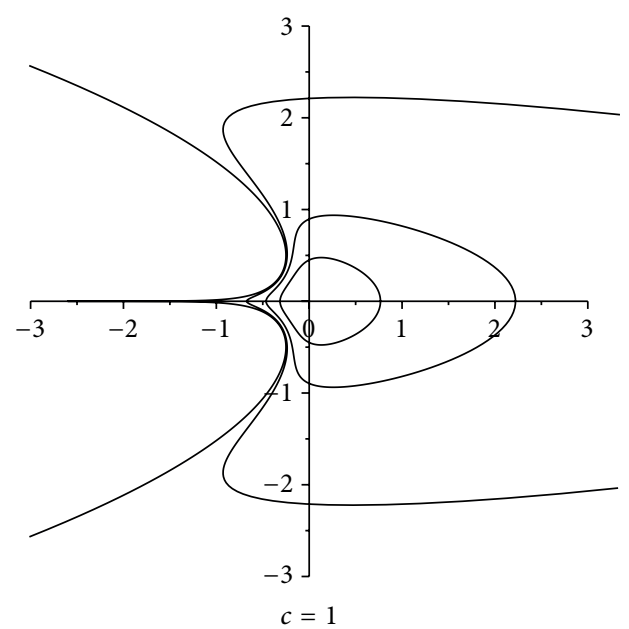

(d)

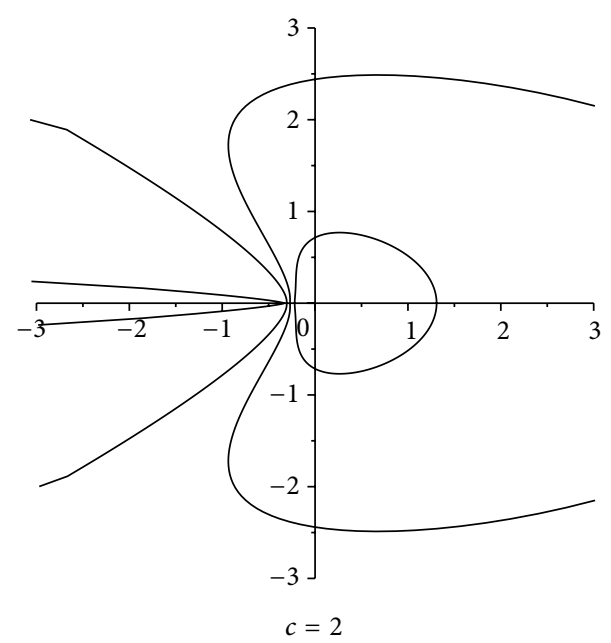

(f)

Figure 1: Images of $f_{c}(|z|=r), r=0.4,0.6,0.8$, and 0.9999 . 


$$
\begin{aligned}
& \mathbf{X}_{1}(z)=( \frac{1}{4} \operatorname{Re}\left(\frac{2 z}{(1-z)^{2}}+\log \frac{1+z}{1-z}\right), \operatorname{Im} \frac{z}{1-z}, \\
&\left.\frac{1}{4} \operatorname{Im}\left(\frac{2 z}{(1-z)^{2}}-\log \frac{1+z}{1-z}\right)\right) \\
& \mathbf{X}_{2}(z)= \frac{1}{3} \operatorname{Re} \frac{z\left(2 z^{2}-3 z+3\right)}{(1-z)^{3}}, \operatorname{Im} \frac{z}{(1-z)^{2}}, \\
&\left.\frac{1}{3} \operatorname{Im} \frac{z^{2}(z-3)}{(1-z)^{3}}\right)
\end{aligned}
$$

which are hardly recognizable as representations of part of the helicoid, wavy plane, and Enneper's surface, respectively. However, as in the proof of Theorem 3, applying the change of variables $w=(1+z) /(1-z)$ and again writing $z=(w-$ $1) /(w+1)$ these representations can be expressed as

$$
\mathbf{X}_{0}(z)=\left(\frac{1}{4} \operatorname{Re}\left(w-\frac{1}{w}\right), \frac{1}{2} \operatorname{Im}(\log w), \frac{1}{4} \operatorname{Im}\left(w+\frac{1}{w}\right)\right)
$$

$$
\begin{aligned}
\mathbf{X}_{1}(z)= & \frac{1}{8} \operatorname{Re}\left(w^{2}+2 \log w-1\right), \frac{1}{2} \operatorname{Im} w \\
& \left.\frac{1}{4} \operatorname{Im}\left(\frac{1}{2} w^{2}-\log w\right)\right), \\
\mathbf{X}_{2}(z)= & \frac{1}{4} \operatorname{Re}\left(\frac{1}{3} w^{3}+w-\frac{4}{3}\right), \frac{1}{4} \operatorname{Im} w^{2}, \\
& \left.\frac{1}{4} \operatorname{Im}\left(\frac{1}{3} w^{3}-w\right)\right) .
\end{aligned}
$$

Comparing the transformed surface given in (16) with the parameterization of the helicoid in (2), we see that interchanging the second and third coordinates of $\mathbf{X}_{0}$ in (16) and scaling by a factor of 4 give the formula for the helicoid given in (2). Since the formula in (2) extends into the right half plane, we see that $\mathbf{X}_{0}(\mathbb{D})$ is the same surface as $\mathbf{Y}_{0}(\{z \in \mathbb{C}$ : $\operatorname{Re} z>0\})$. That is, $\mathbf{X}_{0}(\mathbb{D})$ is part of the helicoid. Similarly, interchanging the second and third coordinates of $\mathbf{X}_{1}$ given in (17), scaling by a factor of 2 , and a translation by a constant, we have the conjugate surface to $Y_{1}$ given in (3). Since the conjugate surface of the wavy plane is itself, we see that $\mathbf{X}_{1}(\mathbb{D})$ is the same surface as $\mathbf{Y}_{1}(\{z \in \mathbb{C}: \operatorname{Re} z>0\})$. Hence, $\mathbf{X}_{1}(\mathbb{D})$ is part of the wavy plane. Finally, using the same approach as done for $\mathbf{X}_{1}, \mathbf{X}_{2}(\mathbb{D})$ is the same surface as $\mathbf{Y}_{2}(\{z \in \mathbb{C}: \operatorname{Re} z>$ $0\})$, and so $\mathbf{X}_{2}(\mathbb{D})$ is part of the Enneper surface. See Figure 2 for an image of the surfaces $\mathbf{X}_{0}, \mathbf{X}_{1}$, and $\mathbf{X}_{2}$.

In general, for all $c \in[0,2], f_{c}=h_{c}+\overline{g_{c}}$ given in (9) lift to a minimal graph, and for $c \neq 0,1$ and $z=(w-1) /(w+1)$ this graph is given by

$$
\begin{aligned}
\mathbf{X}_{c}(z)= & \left(\frac{1}{4\left(c^{2}-1\right)} \operatorname{Re}\left((c-1) w^{c+1}+(c+1) w^{c-1}-2 c\right),\right. \\
& \frac{1}{2 c} \operatorname{Im}\left(w^{c}-1\right) \\
& \left.\frac{1}{4\left(c^{2}-1\right)} \operatorname{Im}\left((c-1) w^{c+1}-(c+1) w^{c-1}\right)\right)
\end{aligned}
$$

Thus, as the parameter $c$ increases from 0 to 2 , the minimal graphs transform continuously from the helicoid to the wavy plane and finally to the Enneper surface.

It is now evident that the surfaces constructed by Hengartner and Schober in [5] to obtain sharp bounds for curvature estimates are indeed related to those in Theorem 4. That is, the surface lying over the entire half-plane given by Hengartner and Schober is the conjugate to the surface given by $\mathbf{X}_{1}$ in (14). As the wavy plane is conjugate to itself, we have now identified the surface in [5] as the wavy plane. Further, for the surface lying over the slit plane with a slit lying on the real axis constructed in [5], we now see it as a polynomial Enneper surface through the same change of variables and similar analysis as above.

With the results from Theorem 4 on the nature of the surface $\mathbf{X}_{c}, c \in[0,2]$, formed by lifting $f_{c}$ as given by (9) in Theorem 3 , we use the following theorem which provides a connection between harmonic mappings, conjugate surfaces, and associated surfaces to provide the two-parameter family of minimal graphs as described in the introduction.

Theorem 5. Let $\varphi \in S$ with $\varphi(\mathbb{D})$ convex and $b \in \mathscr{H}_{0}(\mathbb{D})$. Define the harmonic function $f: \mathbb{D} \rightarrow \mathbb{C}$ such that $f=h+\bar{g}$ satisfies $h-g=\varphi, g^{\prime} / h^{\prime}=b^{2}, h(0)=g(0)=g^{\prime}(0)=0$, and $h^{\prime}(0)=1$. For $\theta \in \mathbb{R}$, define $f_{\theta}: \mathbb{D} \rightarrow \mathbb{C}$ to be $f_{\theta}=$ $h_{\theta}+\overline{g_{\theta}}=e^{i \theta / 2} h+\overline{e^{i \theta / 2} g}$. Then $f_{\theta}$ lifts to a minimal graph $\mathbf{X}_{\theta}$ on $\mathbb{D}$. Moreover, $\mathbf{X}_{0}$ and $\mathbf{X}_{\pi}$ are conjugate surfaces and for each $\theta \in(0, \pi), \mathbf{X}_{\theta}$ is an associated surface of $\mathbf{X}_{0}$.

Proof. First, observe by Theorem 1, $f \in S_{H}^{0}$ and maps $\mathbb{D}$ onto a domain convex in the direction of the real axis. Second, for $\alpha=\pi-\theta / 2, h-e^{2 i \alpha} e^{i \theta} g=\varphi$ and the dilatation of $e^{-i \theta / 2} f_{\theta}=h+$ $\overline{e^{i \theta} g}$ is $e^{i \theta} b^{2}$. Thus, by this same theorem, we see that $e^{-i \theta / 2} f_{\theta} \in$ $S_{H}^{0}$ and maps $\mathbb{D}$ onto domain convex in the direction of $\alpha=$ $\pi-\theta / 2$.

Since $f_{\theta}$ is simply a rotation of a univalent harmonic function, by Theorem 2 , it is clear that $f_{\theta}$ lifts to a minimal graph $\mathbf{X}_{\theta}$. Moreover, for $\theta \in[0, \pi]$ and $z \in \mathbb{D}$,

$$
\begin{aligned}
\mathbf{X}_{\theta}(z)= & \left(\operatorname{Re}\left(e^{i \theta / 2}(h(z)+g(z))\right), \operatorname{Im}\left(e^{i \theta / 2}(h(z)-g(z))\right)\right. \\
& \left.2 \operatorname{Im}\left(e^{i \theta / 2} \int_{0}^{\zeta} \sqrt{h^{\prime}(\zeta) g^{\prime}(\zeta)} d \zeta\right)\right) \\
= & \left(\cos \frac{\theta}{2}\right)(\operatorname{Re}(h(z)+g(z)), \operatorname{Im}(h(z)-g(z)) \\
& +\left(\sin \frac{\theta}{2}\right)(-\operatorname{Im}(h(z)+g(z)), \operatorname{Re}(h(z)-g(z)) \\
= & \left(\cos \frac{\theta}{2}\right)(\operatorname{Re}(h(z)+g(z)), \operatorname{Im}(h(z)-g(z)) \\
& \left.\quad 2 \operatorname{Re}\left(\int_{0}^{z} \sqrt{h^{\prime}(\zeta) g^{\prime}(\zeta)} d \zeta\right)\right) \\
& \left.\quad \operatorname{Im}\left(\int_{0}^{z} \sqrt{h^{\prime}(\zeta) g^{\prime}(\zeta)} d \zeta\right)\right)
\end{aligned}
$$




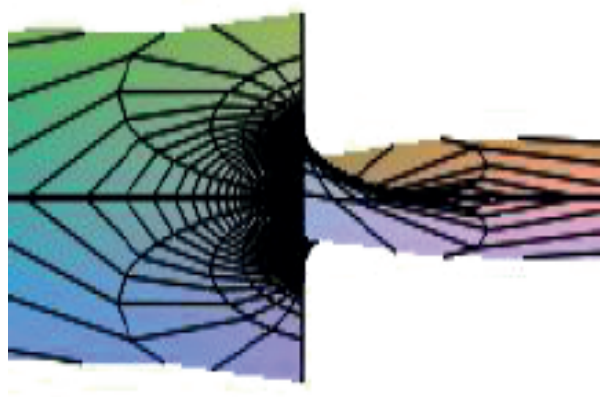

$X_{0}(\mathbb{D})$

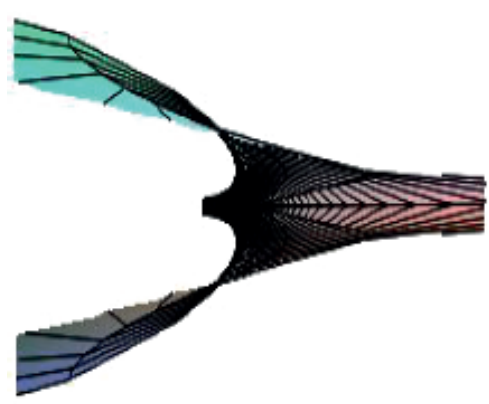

$X_{1}(\mathbb{D})$

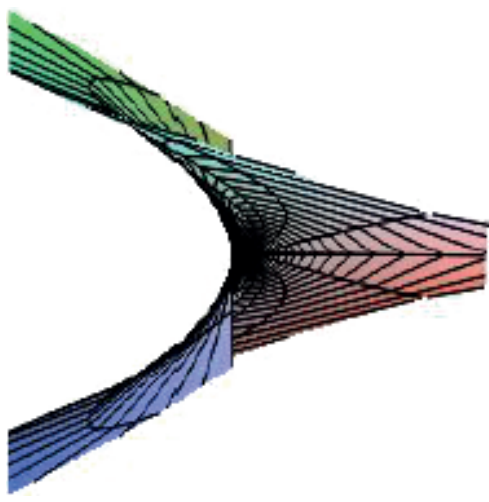

$X_{2}(\mathbb{D})$

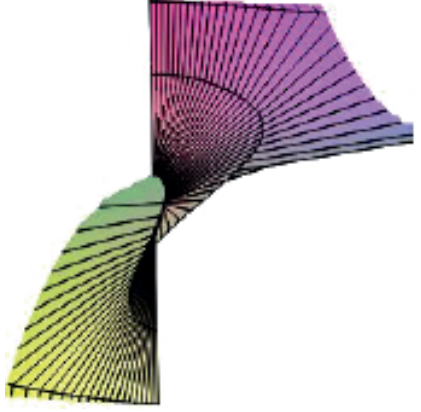

$X_{0}(\{z: \operatorname{Re} z<0\})$

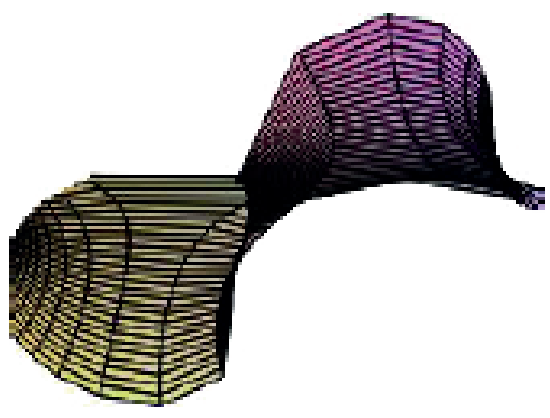

$X_{1}(\{z: \operatorname{Re} z<0\})$

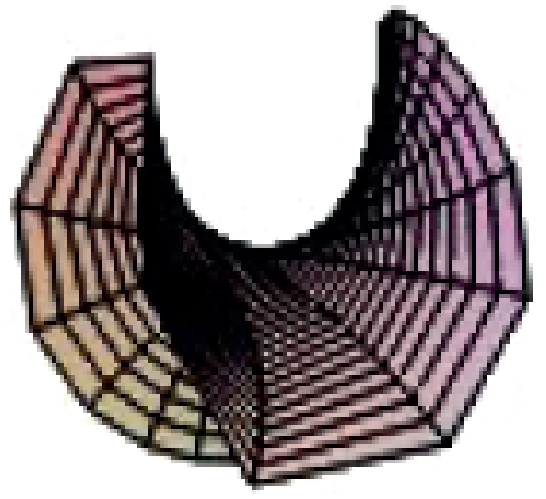

$X_{2}(\{z: \operatorname{Re} z<0\})$

FIGURE 2: Images of $\mathbf{X}_{c}$.

$$
\begin{aligned}
&+\left(\sin \frac{\theta}{2}\right)( \operatorname{Re}(i(h(z)+g(z))), \\
& \operatorname{Im}(i(h(z)-g(z))), \\
&\left.2 \operatorname{Im}\left(i \int_{0}^{z} \sqrt{h^{\prime}(\zeta) g^{\prime}(\zeta)} d \zeta\right)\right) \\
&=\left(\cos \frac{\theta}{2}\right) \mathbf{X}_{0}(z)+\left(\sin \frac{\theta}{2}\right) \mathbf{X}_{\pi}(z) .
\end{aligned}
$$

Thus, what remains to be shown is that $\mathbf{X}_{0}$ and $\mathbf{X}_{\pi}$ are conjugate surfaces. Using line (20), we see that the coordinate functions of $\mathbf{X}_{0}$ are the imaginary parts of the analytic functions $i(h+g), h-g$, and $\int_{0}^{z} \sqrt{h^{\prime}(\zeta) g^{\prime}(\zeta)} d \zeta=\int_{0}^{z} h^{\prime}(\zeta) b(\zeta) d \zeta$, respectively, while the coordinate functions of $\mathbf{X}_{\pi}$ are the real parts, respectively. Therefore, $\mathbf{X}_{0}$ and $\mathbf{X}_{\pi}$ satisfy the CauchyRiemann equations and are conjugate surfaces.

Notice that in the proof of Theorem 5, we have shown that if $f=h+\bar{g}$ lifts to a minimal graph on $\mathbb{D}$ and $(h-g)(\mathbb{D})$ is convex, then $F=i h+\overline{i g}$ lifts to the conjugate minimal graph on $\mathbb{D}$. By a similar approach, we see that for any $\theta \in \mathbb{R}$, if $f_{\theta}$ on $\mathbb{D}$ lifts to a minimal graph and $\left(h_{\theta}-g_{\theta}\right)(\mathbb{D})$ is convex, then $f_{\theta+\pi}$ on $\mathbb{D}$ will lift to its conjugate minimal graph.

Corollary 6. For $c \in[0,1]$ and $\theta \in[0, \pi]$, define $f_{c, \theta}: \mathbb{D} \rightarrow$ $\mathbb{C}$ by

$$
f_{c, \theta}(z)=h_{c, \theta}(z)+\overline{g_{c, \theta}(z)}=e^{i \theta / 2} h_{c}(z)+\overline{e^{i \theta / 2} g_{c}(z)},
$$

where $h_{c}-g_{c}$ is defined by (9) and is normalized by $h_{c}(0)=$ $g_{c}(0)=g_{c}^{\prime}(0)=h_{c}^{\prime}(0)-1=0$. Then for each $c \in[0,1]$ 
TABLE 1: Images of $f_{c, \theta}(|z|=r), r=0.4,0.6,0.8,0.9999$, and the corresponding minimal surfaces.

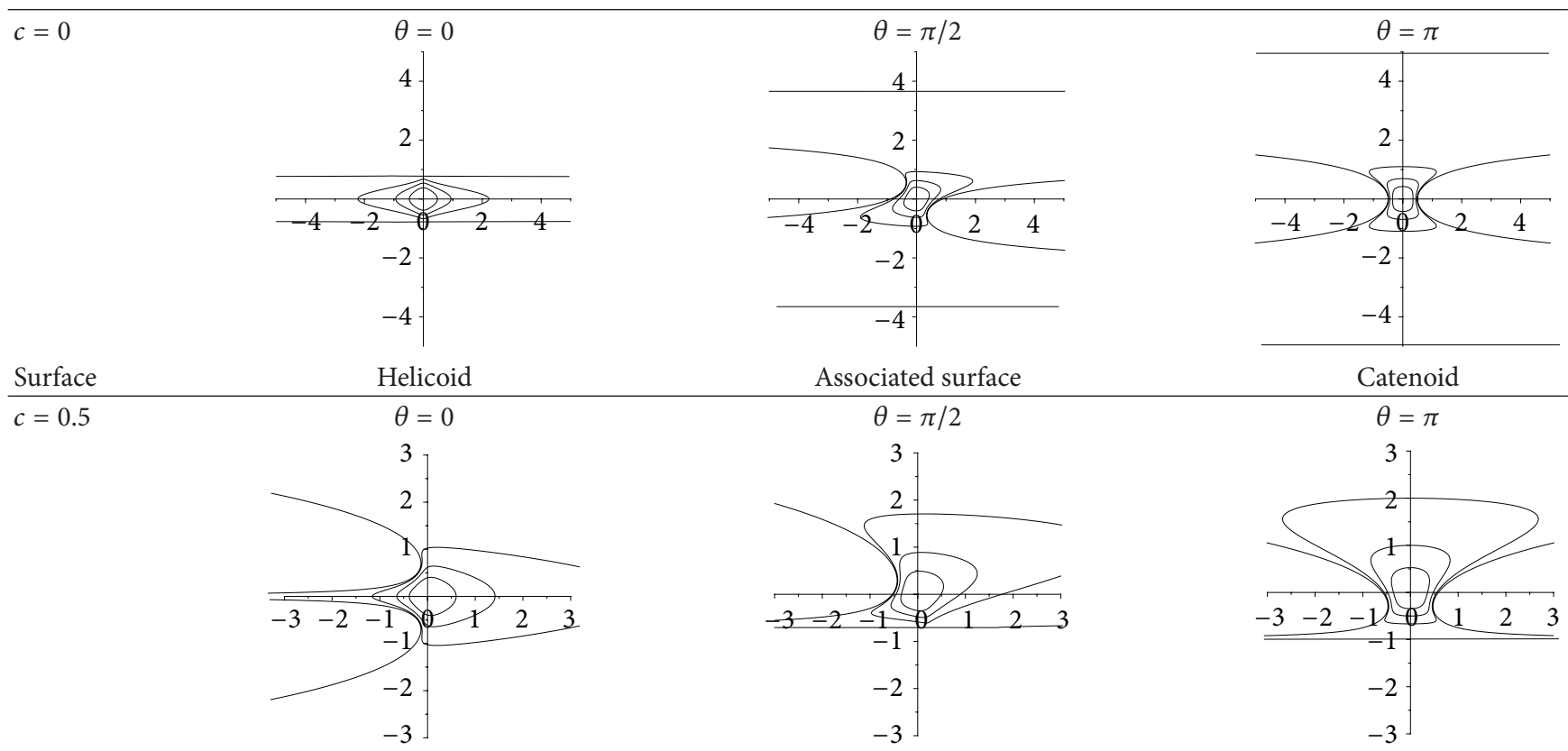

Surface
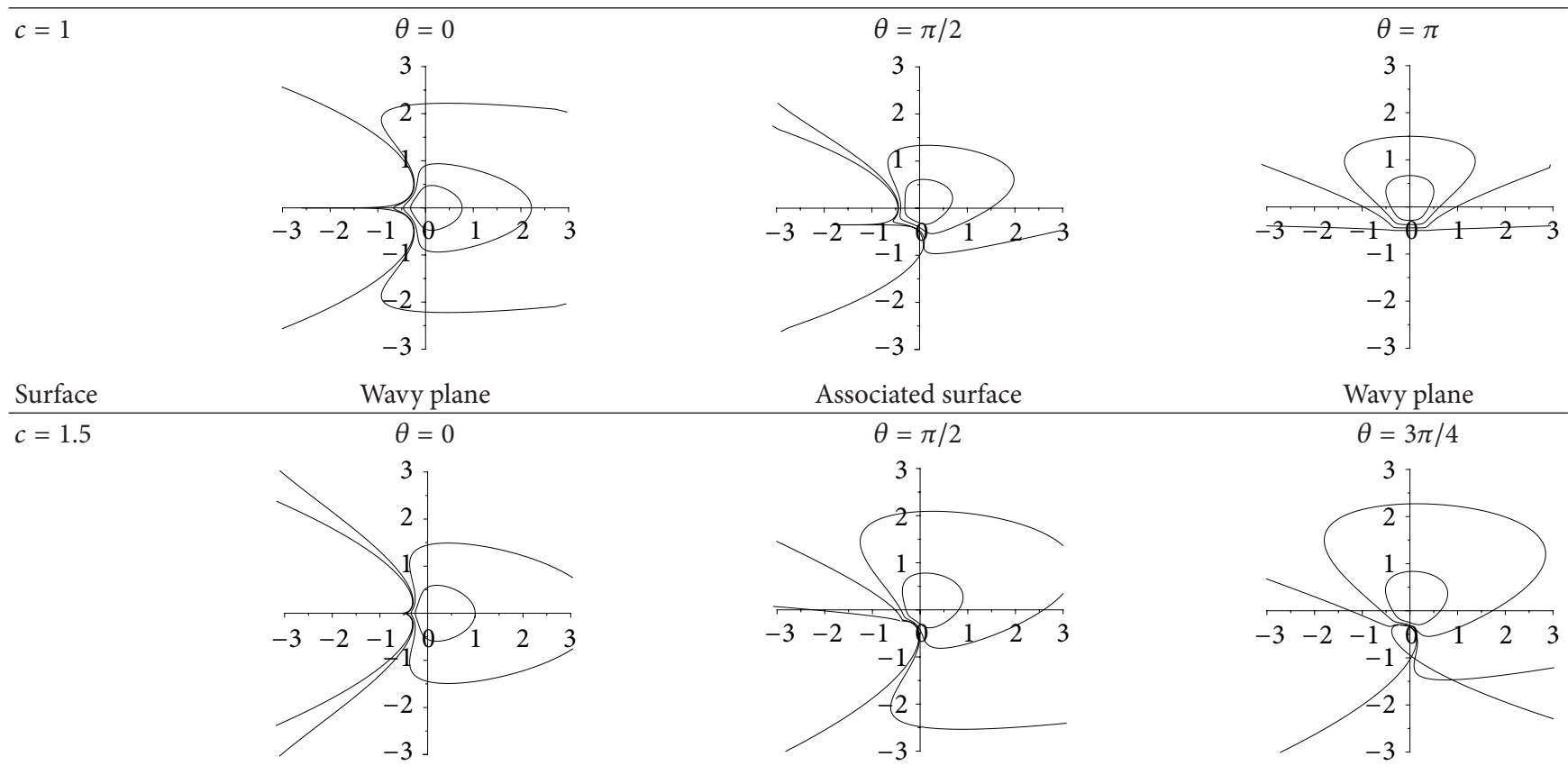

Associated surface

Wavy plane

Surface
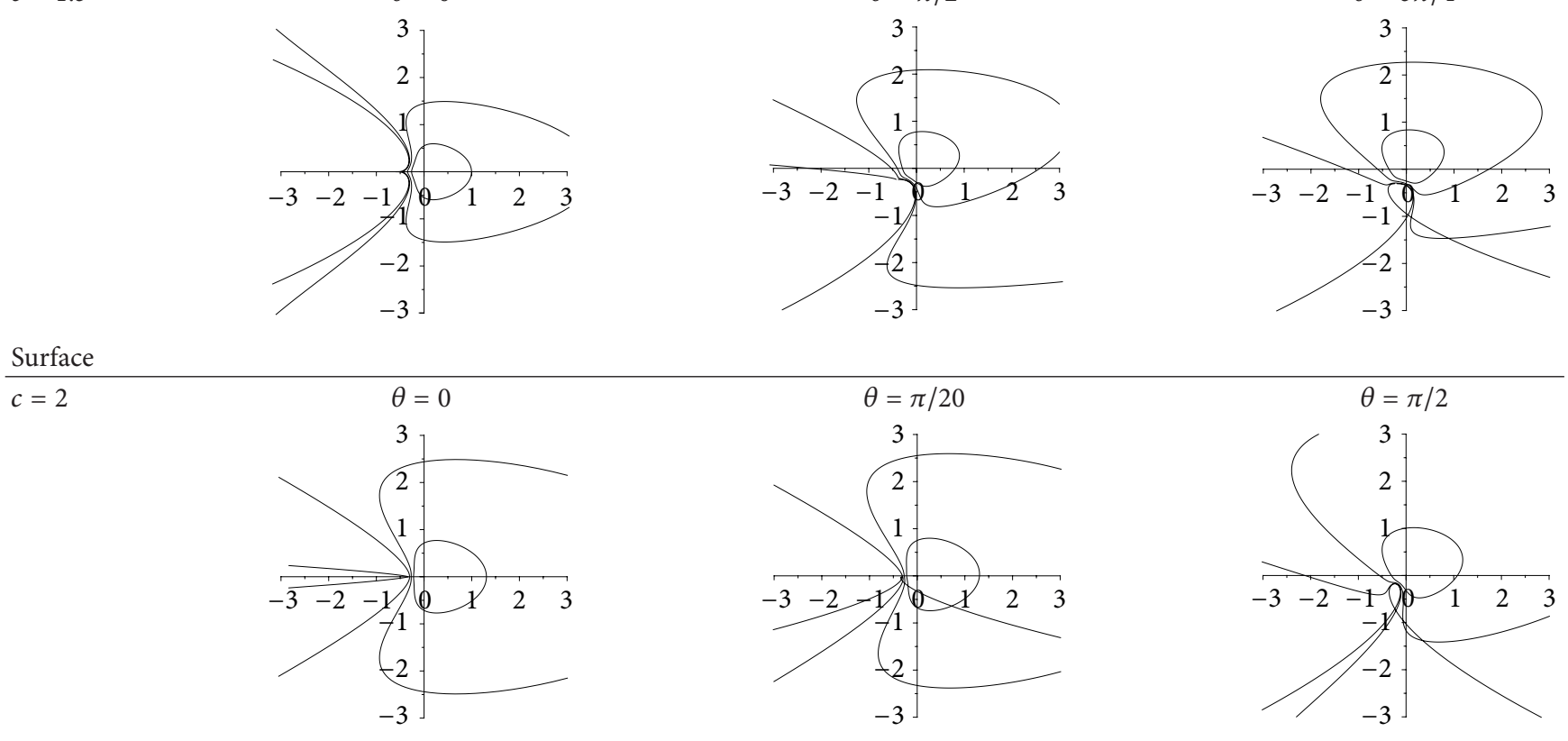
and $\theta \in[0, \pi], f_{c, \theta}$ lifts to a minimal graph $\mathbf{X}_{c, \theta}$, and for each $c \in[0,1], \mathbf{X}_{c, 0}$ and $\mathbf{X}_{c, \pi}$ are conjugate surfaces.

Proof. For $c \in[0,1]$ and $\theta \in[0, \pi]$, let $f_{c, \theta}: \mathbb{D} \rightarrow \mathbb{C}$ be defined as in (21). Recall that, for $c \in[0,1]$ and $k_{c}$ given by $(7), k_{c}(\mathbb{D})$ is convex. This in conjunction with $(9)$ and Theorem 5 allows for the construction of a two-parameter family of minimal graphs $\mathbf{X}_{c, \theta}$ using the Weierstrass-Enneper representation of Theorem 2 to lift $f_{c, \theta}$. For a fixed $c \in[0,1]$, $\mathbf{X}_{c, 0}$ is given by the surfaces $\mathbf{X}_{c}$ in (13), (14), and (19). Thus, for a fixed $c \in[0,1]$, as $\theta$ ranges from 0 to $\pi$, the surface $\mathbf{X}_{c, \theta}$ transforms from associated surfaces of $\mathbf{X}_{c}$ given in (13), (14), and (19) to the respective conjugate surface.

\section{Problems for Further Investigation}

(1) As noted earlier, Theorem 2 states that a univalent harmonic map lifts to a minimal surface that is a graph. Nonetheless, a harmonic map with a perfect square dilatation always lifts to a minimal surface. There is just no guarantee that the surface is a graph over its domain. Thus, while we can only be certain for $c \in[0,1]$ that as $\theta$ varies in $[0, \pi], f_{c, \theta}$ given in Corollary 6 lifts to a minimal graph, we conjecture that for $c \in(1,2]$ there exists some $\theta_{c}<\pi$ for which $f_{c, \theta}, \theta \in\left(0, \theta_{c}\right)$, is univalent and hence would lift to a minimal graph by Theorem 2. Further, it appears that as $c$ increases towards two, $\theta_{c}$ decreases towards zero. Table 1 summarizes some information relating the images of $f_{c, \theta}(\mathbb{D})$ and the corresponding minimal surfaces for various values of $c$ and $\theta$ providing some evidence in support of the conjectures for $c \in(1,2]$.

(2) Additionally, the family of harmonic mappings given in Theorem 3 can also be generalized by changing the dilatation to $\omega(z)=z^{2 n}, n \in \mathbb{N}$. That is, for $c \in[0,2]$ and $n \in \mathbb{N}$, let $f_{c, n}=h_{c, n}+\overline{g_{c, n}}: \mathbb{D} \rightarrow \mathbb{C}$, where

$$
h_{c, n}(z)-g_{c, n}(z)=k_{c}(z), \quad g_{c, n}^{\prime}(z)=z^{2 n} h_{c, n}^{\prime}(z) \text {. }
$$

The case $n=1$ is the basis of this paper. For the case $n=2$ and $c=2$, it appears that the resulting minimal surface is a helicoid. It would be interesting to use the techniques from this paper to investigate the family of functions $f_{c, n}$ for $n \geq 2$.

\section{Conflict of Interests}

The authors declare that there is no conflict of interests regarding the publication of this paper.

\section{Acknowledgments}

This paper is an outgrowth of initial examples investigated by Amanda Curtis and Rachel Messick during the 2010 BYU REU supported by the NSF Grant DMS-0755422. The second author thanks BYU for support as a Visiting Scholar in the spring of 2011.

\section{References}

[1] M. Dorff, "Minimal graphs in $R^{3}$ over convex domains," Proceedings of the American Mathematical Society, vol. 132, no. 2, pp. 491-498, 2004.

[2] M. Dorff and J. Szynal, "Harmonic shears of elliptic integrals," The Rocky Mountain Journal of Mathematics, vol. 35, no. 2, pp. 485-499, 2005.

[3] K. Driver and P. Duren, "Harmonic shears of regular polygons by hypergeometric functions," Journal of Mathematical Analysis and Applications, vol. 239, no. 1, pp. 72-84, 1999.

[4] P. Duren and W. Thygerson, "Harmonic mappings related to Scherk's saddle-tower minimal surfaces," The Rocky Mountain Journal of Mathematics, vol. 30, no. 2, pp. 555-564, 2000.

[5] W. Hengartner and G. Schober, "Curvature estimates for some minimal surfaces," in Complex Analysis, pp. 87-100, Birkhäuser, Basel, Switzerland, 1988.

[6] J. McDougall and L. Schaubroeck, "Minimal surfaces over stars," Journal of Mathematical Analysis and Applications, vol. 340, no. 1, pp. 721-738, 2008.

[7] M. Nowak and M. Wołoszkiewicz, "Gauss curvature estimates for minimal graphs," Annales Universitatis Mariae CurieSkłodowska A, vol. 65, no. 2, pp. 113-120, 2011.

[8] J. Clunie and T. Sheil-Small, "Harmonic univalent functions," Annales Academiae Scientiarum Fennicae A, vol. 9, pp. 3-25, 1984.

[9] H. Lewy, "On the non-vanishing of the Jacobian in certain one-to-one mappings," Bulletin of the American Mathematical Society, vol. 42, no. 10, pp. 689-692, 1936.

[10] U. Dierkes, S. Hildebrandt, A. Küster, and O. Wohlrab, Minimal Surfaces I, vol. 295 of Grundlehren der Mathematischen Wissenschaften, Springer, Berlin, Germany, 1992.

[11] M. Dorff, "Soap films, differential geometry, and minimal surfaces," in Explorations in Complex Analysis, pp. 85-159, Mathematical Association of America, Washington, DC, USA, 2012.

[12] M. Weber, "Classical minimal surfaces in Euclidean space by examples: geometric and computational aspects of the Weierstrass representation," in Global Theory of Minimal Surfaces, vol. 2 of Clay Mathematics Proceedings, pp. 19-63, Mathematical Association of America, Providence, RI, USA, 2005.

[13] P. Duren, Harmonic Mappings in the Plane, vol. 156 of Cambridge Tracts in Mathematics, Cambridge University Press, Cambridge, UK, 2004. 


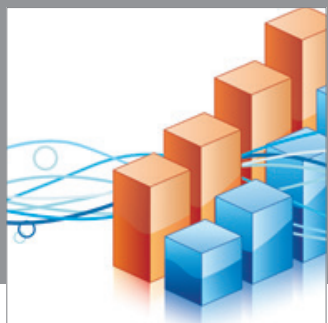

Advances in

Operations Research

mansans

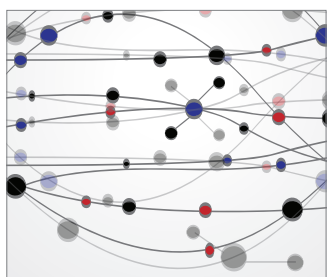

The Scientific World Journal
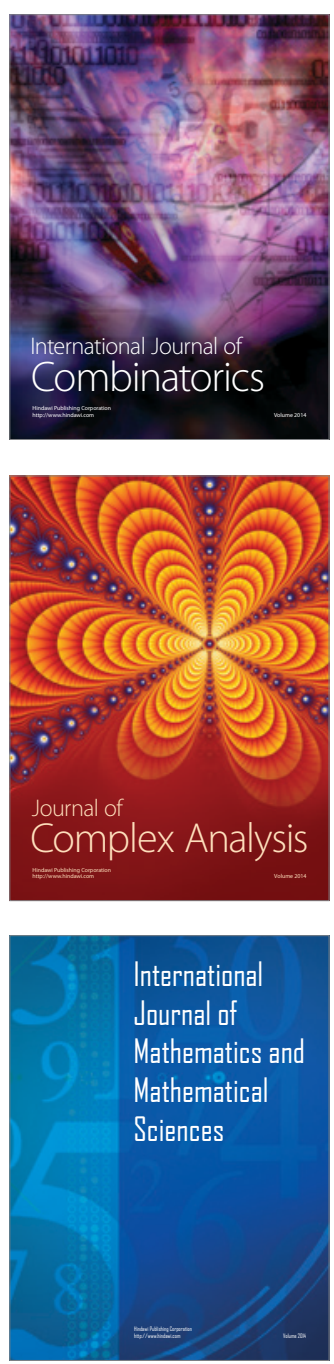
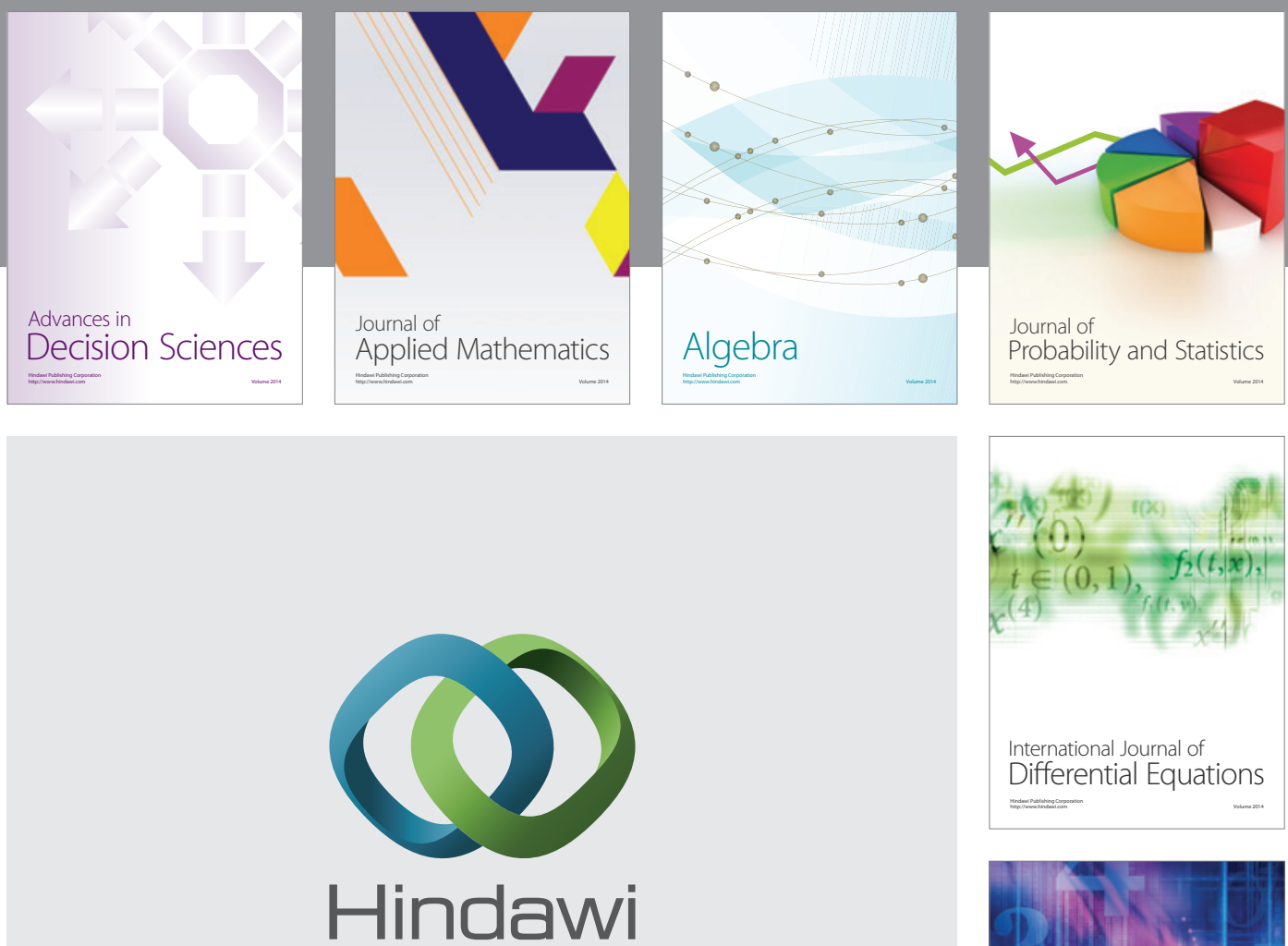

Submit your manuscripts at http://www.hindawi.com
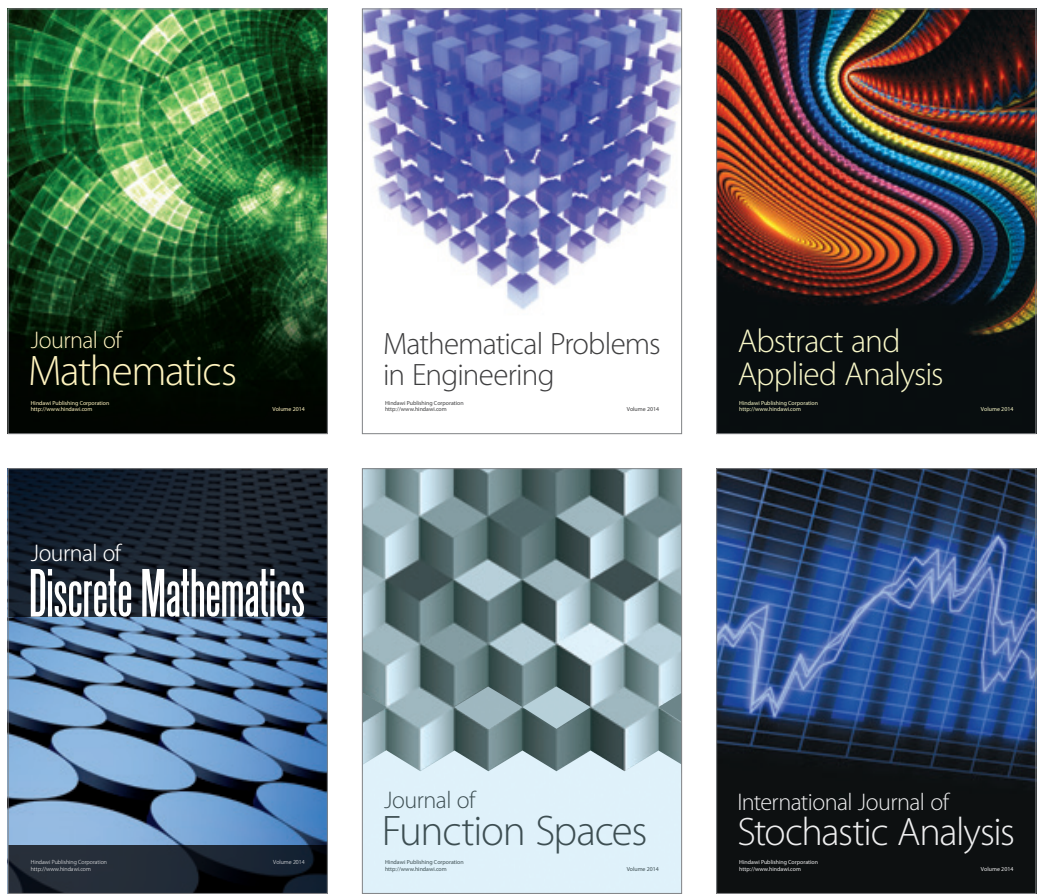

Journal of

Function Spaces

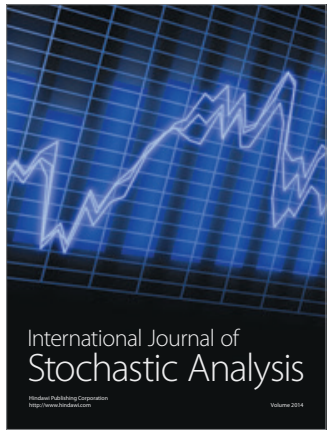

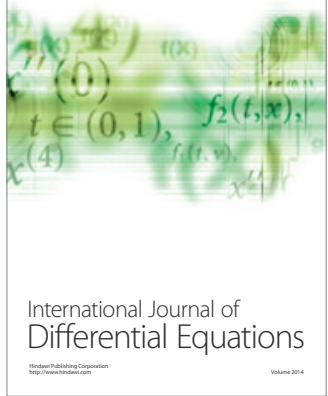
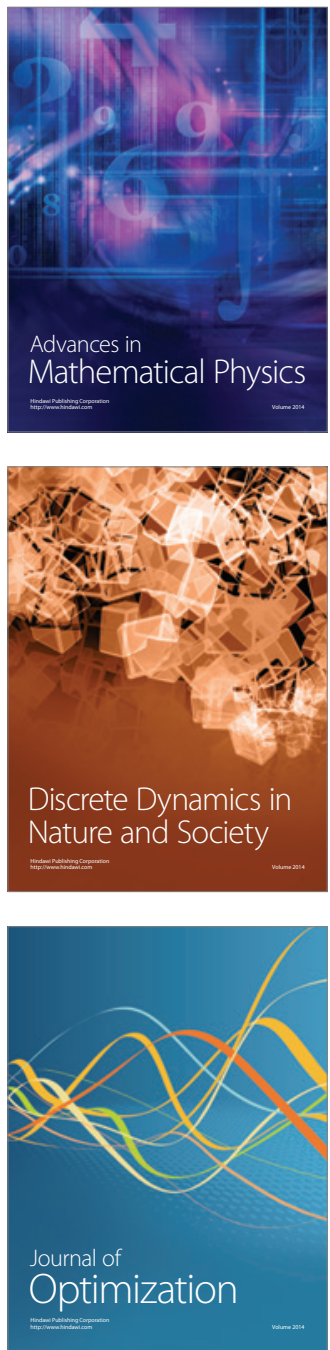